\title{
Artificial Fish Swarm Algorithm-Based Particle Filter for Li-Ion Battery Life Prediction
}

\author{
Ye Tian, ${ }^{1,2}$ Chen Lu, ${ }^{1,2}$ Zili Wang, ${ }^{1,2}$ and Laifa Tao ${ }^{1,2}$ \\ ${ }^{1}$ School of Reliability and Systems Engineering, Beihang University, Beijing 100191, China \\ ${ }^{2}$ Science \& Technology on Reliability \& Environmental Engineering Laboratory, Beijing 100191, China \\ Correspondence should be addressed to Chen Lu; luchen@buaa.edu.cn
}

Received 9 April 2014; Revised 10 July 2014; Accepted 14 July 2014; Published 23 July 2014

Academic Editor: Qingsong Xu

Copyright (c) 2014 Ye Tian et al. This is an open access article distributed under the Creative Commons Attribution License, which permits unrestricted use, distribution, and reproduction in any medium, provided the original work is properly cited.

\begin{abstract}
An intelligent online prognostic approach is proposed for predicting the remaining useful life (RUL) of lithium-ion (Li-ion) batteries based on artificial fish swarm algorithm (AFSA) and particle filter (PF), which is an integrated approach combining model-based method with data-driven method. The parameters, used in the empirical model which is based on the capacity fade trends of Li-ion batteries, are identified dependent on the tracking ability of PF. AFSA-PF aims to improve the performance of the basic PF. By driving the prior particles to the domain with high likelihood, AFSA-PF allows global optimization, prevents particle degeneracy, thereby improving particle distribution and increasing prediction accuracy and algorithm convergence. Data provided by NASA are used to verify this approach and compare it with basic PF and regularized PF. AFSA-PF is shown to be more accurate and precise.
\end{abstract}

\section{Introduction}

In recent years, green energy has gained increasing support among the people and electric energy has become much more popular. Lithium-ion (Li-ion) batteries offer advantages such as a high energy ratio, high voltage, desirable cryogenic properties, low self-discharge ratio, and lack of the memory effect [1]. Li-ion batteries are therefore widely used in electric vehicles, satellites, aerospace craft, and other important domains. Being the core cell of several types of electronic equipment and complex systems [2], Li-ion batteries are a critical component of the electronic system as a whole, and the failure of $\mathrm{Li}$-ion batteries can lead to system malfunction, downed systems, and even human casualties and financial losses [3].

The capacity of Li-ion batteries inevitably degrades over time. Hence, monitoring the state of health of $\mathrm{Li}$-ion batteries in operation is of great necessity. The monitored data can be used to predict the remaining useful life (RUL) of Liion batteries, which can provide the basis for maintenance decisions and make the use of Li-ion batteries much more convenient and safer. Currently, the optimal prediction of the
RUL of Li-ion batteries has aroused intense discussion in the Prognostic and Health Management (PHM) domain locally and abroad $[1,4]$.

Several studies have focused on predicting the RUL of Liion batteries [5-7]. Zhang and Lee reviewed various aspects of recent research and developments in Li-ion battery PHM and summarized the techniques, algorithms, and models used in RUL prediction [8]. Regarding research on methods of forecasting the RUL of Li-ion batteries, Saha et al. from the Prognostics Center of Excellence (PCoE) of NASA were first to develop a number of performance degradation tests for Li-ion batteries under various test conditions [9]. The parameters measured were comprehensive. The researchers obtained a large amount of test data and took the lead in using the Bayesian estimation method to forecast the RUL of Li-ion batteries. The core idea of this method is to build a probability density distribution (PDF) of states based on all available information [10] to establish a way to express and manage the uncertainty of prediction. The researchers forecast the RUL of Li-ion batteries by establishing a particle filter- (PF-) based method. Later, Saha et al. proposed a forecasting method combining support vector machine with 
PF on the basis of [10]. This method was able to calculate the PDF of the prediction value, rather than mean time to failure, accurately and precisely.

Given the complexity of the system organization of Li-ion batteries, the appropriate operating conditions are changeable and the system often suffers from noise jamming, test data, model error, load variation, operating conditions, and other uncertain factors need to be taken into consideration [10]. $\mathrm{PF}$ is an effective solution for these problems in PHM for nonlinear non-Gaussian systems. PF is able to dynamically adjust model parameters of nonstationary conditions and predict the unknown parameters of the battery empirical model by tracking historical data. Accurate prediction as well as a confidence evaluation of the prediction value can be obtained by means of PF. The estimation of confidence interval is often characterized by PDF. PF describes the posteriori estimate of a state using a set of particles with weight; this method results in a probability expression for the prediction value. This description, which is based on Monte Carlo, is equivalent to a real posterior probability density function, and effectively expresses the uncertainty of the prediction outcome, thereby making PF an ideal method for state tracking and prediction [11].

However, basic PF has a serious problem of particle degeneracy [12]. Addressing this problem improperly results in "particle collapse," a severe case of sample impoverishment [13] where the weights of particles accumulate on a small fraction of particles after a few iterations, leaving a poor representation of posterior density. Unfortunately, a number of papers point out that the degeneracy phenomenon is unavoidable. Many improved algorithms have been proposed to tackle this degeneracy problem. All algorithms can be divided into two broad categories: those involving an appropriate choice of importance sampling density or those involving modifying the resampling step [13-17]. In [17], a regularized PF (RPF) approach was applied to estimate system state and predict battery life, proving the ability of this approach to solve the degeneracy problem by obtaining more accurate and more reliable state estimation. In [18], an improved PF based on the artificial fish swarm algorithm (AFSA) was proposed. This algorithm drives prior particles to a high-likelihood domain, allows global optimization, and prevents particle degeneracy to improve particle distribution and increase prediction accuracy and algorithm convergence [19]. In the present study, which aims to achieve higher accuracy and precision, we use AFSA-PF to predict the RUL of Li-ion batteries. Data provided by NASA are used to verify this approach and compare it with basic PF and RPF.

\section{Methodologies}

2.1. Artificial Fish Swarm Algorithm. Artificial fish swarm algorithm (AFSA) was proposed by Li et al. [20] in 2002 as a new bionic random searching optimization algorithm based on simulating the ecological behaviors of fish swarm in the wild. This algorithm mainly imitates four behaviors of fish swarm, that is, preying behavior to find the optimal solution, swarming behavior to gather the fish swarm in the optimal region, following behavior to free the fish swarm from local optimal solutions, and random behavior to find the optimal solution in a larger scope. With the merits of rapid and global optimization, insensitivity to initial values and selections of parameters, robustness, easy operation, and so on, AFSA has been applied widely in pattern recognition, neural networks, and parameter estimation [21-23]. To illustrate the algorithm clearly and expediently, we create the following rules: the individual state of the artificial fish (AF) is denoted by a vector $X=\left(x_{1}, x_{2}, \ldots, x_{n}\right)$, where $x_{i}(i=1,2, \ldots, n)$ is the variable whose optimal value is to be searched for; the food consistency of the position where the AF is currently located is denoted by $Y=f(X)$, where $Y$ denotes the value of the objective function. The distance between two AFSA individuals is expressed as $d_{i, j}=\left\|X_{i}-X_{j}\right\|, v$ represents the vision distance, $s$ represents the largest step-length of the AF, and $r$ represents a random number ranging from zero to one.

The behaviors are described as follows.

Preying Behavior. Let $X_{i}$ denote the current state of the AF and randomly select a state $X_{j}(1)$ within the scope of perception $\left(d_{i, j}<v\right)$. In this study, we only discuss the maximum problem. If $Y_{i}<Y_{j}, X_{i}$ moves a step toward $X_{j}$; otherwise, we select a state $X_{j}$ randomly again and justify whether it satisfies the above requirement or not. After several attempts, if the requirements still cannot be satisfied, $X_{i}$ moves a step randomly. The following briefly shows the process of the preying behavior:

$$
\begin{gathered}
X_{j}=X_{i}+v \cdot r, \\
\text { If } Y_{i}<Y_{j}, \quad X_{\text {inext }}=X_{i}+r \cdot s \cdot \frac{X_{j}-X_{i}}{\left\|X_{j}-X_{i}\right\|} ;
\end{gathered}
$$$$
\text { Else } X_{\text {inext }}=X_{i}+r \cdot s \text {. }
$$

Swarming Behavior. One stipulation for AF is set in the AFSA; that is, AF always tries to swim toward the center of its adjacent fellow swarm-members and avoids being overcrowded, which directs the swarming behavior. Let $X_{i}$ denote the current state of the AF, $n_{f}$ denote the number of the fellow swarm-members within the scope of perception $\left(d_{i, j}<v\right), X_{c}$ denote the center of the fellows around $X_{i}$, and $Y_{c}$ denote the corresponding food consistency of $X_{c}$. The AF swims naturally as it searches around $n_{f}$ and $X_{c}$, if $Y_{c} / n_{f}>$ $\delta \cdot Y_{i}$, which means that the center of the swarm-members has a considerable amount of food and is not crowded. Thus, the AF moves toward the center; otherwise, it executes the preying behavior. The following briefly shows the process of the behavior of swarming:

$$
\text { If } \frac{Y_{c}}{n_{f}}>\delta \cdot Y_{i}, \quad X_{i \text { next }}=X_{i}+r \cdot s \cdot \frac{X_{c}-X_{i}}{\left\|X_{c}-X_{i}\right\|} ;
$$

Else return (2).

Following Behavior. Let $X_{i}$ denote the current state of the AF, and find $X_{j}$ of which $Y_{j}$ is the largest value within the scope 
of perception $\left(d_{i, j}<v\right)$. If $Y_{j} / n_{f}>\delta \cdot Y_{i}$, this means that the fellow $X_{j}$ has high food consistency and the fish surrounding $X_{j}$ is not very crowded. Thus, $X_{i}$ moves a step toward $X_{j}$; otherwise, it executes the preying behavior.

Random Behavior. In nature, random behavior is the default activity of the preying behavior; that is, the AF moves a step randomly in its field of vision. The behavior seems to be a random behavior; however, in this process, the AF searches for food or fellow swarm-members in a larger scope. A few iterations later, the AF would gather around in some local extrema; then a large number of AF would gather around the area of the extremum which is the comparatively optimal area. In this way, we could easily obtain the global extremum to smoothly achieve the optimal value $[19,20]$.

2.2. Particle Filter. Particle filter (PF) is a statistical filtering technique for implementing the recursive Bayesian filtering using the Monte Carlo simulations [24]. In the PF approach, the state probability density function (PDF) is approximated by a set of particles (points) representing sampled values from an unknown state space and a set of associated weights denoting discrete probability masses. The particles are generated and recursively updated from a nonlinear process model that describes the evolution of the system under analysis in time with a measurement model, a set of available measurements, and an a priori estimate of the state PDF.

$\mathrm{PF}$ methods assume that the state equations can be modeled as a first order Markov process with the outputs being conditionally independent. This model can be written as

$$
\begin{aligned}
& x_{k}=f\left(x_{k-1}\right)+\omega_{k}, \\
& y_{k}=h\left(x_{k}\right)+v_{k},
\end{aligned}
$$

where $x$ denotes the state, $y$ is the output or measurements, and $\omega_{k}$ and $v_{k}$ are samples from a noise distribution, respectively. Sequential importance resampling (SIR) is a widely used particle filtering algorithm, which approximates the filtering distribution denoted as $p\left(x_{k} \mid y_{0}, \ldots, y_{k}\right)$ by a set of $P$ weighted particles $\left\{\left(w_{k}^{i}, x_{k}^{i}\right): i=1, \ldots P\right\}$. The importance weights $w_{k}^{i}$ are approximations to the relative posterior probabilities of the particles such that

$$
\begin{gathered}
\int f\left(x_{k}\right) p\left(x_{k} \mid y_{0}, \ldots, y_{k}\right) d x_{k} \approx \sum_{i=1}^{P} w_{k}^{i} f\left(x_{k}^{i}\right), \\
\sum_{i=1}^{P} w_{k}^{i}=1 .
\end{gathered}
$$

The weight update is given by

$$
w_{k}^{i}=w_{k-1}^{i} \frac{p\left(y_{k} \mid x_{k}\right) p\left(x_{k} \mid x_{k-1}\right)}{\pi\left(x_{k} \mid x_{0: k-1}, y_{1: k}\right)},
$$

where the importance distribution $\pi\left(x_{k} \mid x_{0: k-1}, y_{1: k}\right)$ is approximated as $p\left(x_{k} \mid x_{k-1}\right)$.
The procedure of the basic particle filter is as follows.

(1) Initialization. $k=0$ is set, $N$ particles are sampled and represented as $\left\{x_{0}^{i}, i=1,2, \ldots, N\right\}$ from prior distribution $p\left(x_{0}\right)$, and each weight of the sample is set to $1 / N$ as the initial weight.

(2) Importance Sampling. $k=1$ is set and the state of particles is updated using the importance distribution which can be represented as $x_{k}^{i} \sim \pi\left(x_{k} \mid x_{0: k-1}^{i}, y_{0: k}\right), i=1,2, \ldots, N$.

The importance weights are then calculated according to (6) and the weights are normalized as $w_{k}^{i}=w_{k}^{i} / \sum_{i=1}^{N} w_{k}^{i}$.

(3) Resampling. Ahead of time, a threshold number of effective particles is set as $N_{\text {threshold }}$. The number of effective particles are calculated and then denoted as $N_{\text {eff }}=$ $1 / \sum_{i=1}^{N}\left(w_{k}^{i}\right)^{2}$. If $N_{\text {eff }}<N_{\text {threshold }}$, the particles are resampled; that is, the weighted particles $\left\{x_{0: k}^{i}, w_{k}^{i}\right\}_{i=1}^{N}$ are mapped to become equal to the weighted particles $\left\{x_{0: k}^{i}, N^{-1}\right\}_{i=1}^{N}$.

(4) Prediction. $x_{k+1}^{i}$ is obtained using the state equations (4). We set $k=k+1$ and then proceed to Step $(2)[5,19]$.

The regularized particle filter is an improved version of the basic PF. By means of resampling from a continuous approximation instead of resampling from a discrete approximation of the posterior density $p\left(x_{k} \mid z_{1: k}\right)$; RPF exactly avoids the problem of basic PF. In this study, we do not discuss RPF further; detailed information about RPF could be obtained in $[13,15]$.

2.3. AFSA-Based Particle Filter. Introducing the concept of AFSA into basic PF aims at avoiding the phenomenon of particle degeneracy. The combination is applied in the selection of the objective function and the adjustment of the importance weights. AFSA is introduced to improve the process of importance sampling in the basic particle filter by driving the particles move to the high likelihood areas. In this study, we consider the objective function as the posterior PDF. We refer to the new method as AFSA-PF. In AFSA-PF, the number of fish is the same as the number of particles.

Likelihood function is defined as the objective function $Y$, which is also the posterior PDF:

$$
p\left(y_{k}^{i} \mid x_{k}^{i}\right)=\frac{1}{\left(2 \pi \sigma_{v}{ }^{2}\right)^{1 / 2}} \exp \left[-\frac{1}{2 \sigma_{v}{ }^{2}}\left(y_{k}-y_{k \mid k-1}^{i}\right)^{2}\right],
$$

where $y_{k}$ is the latest observed value and $y_{k \mid k-1}^{i}$ is the prediction of the observed value of the last moment.

The main process of AFSA-PF is based on the process of $\mathrm{PF}$ as described in Section 2.2. The difference between AFSA-PF and the basic PF lies in importance sampling, as shown in Figure 1. The detailed process of AFSA-PF is described as follows.

(1) Parameter Initialization. The initial states of particles and some parameters (number of particles, process noise, measurement noise, etc.) need to be determined, similar to Step (1) in Section 2.2. 


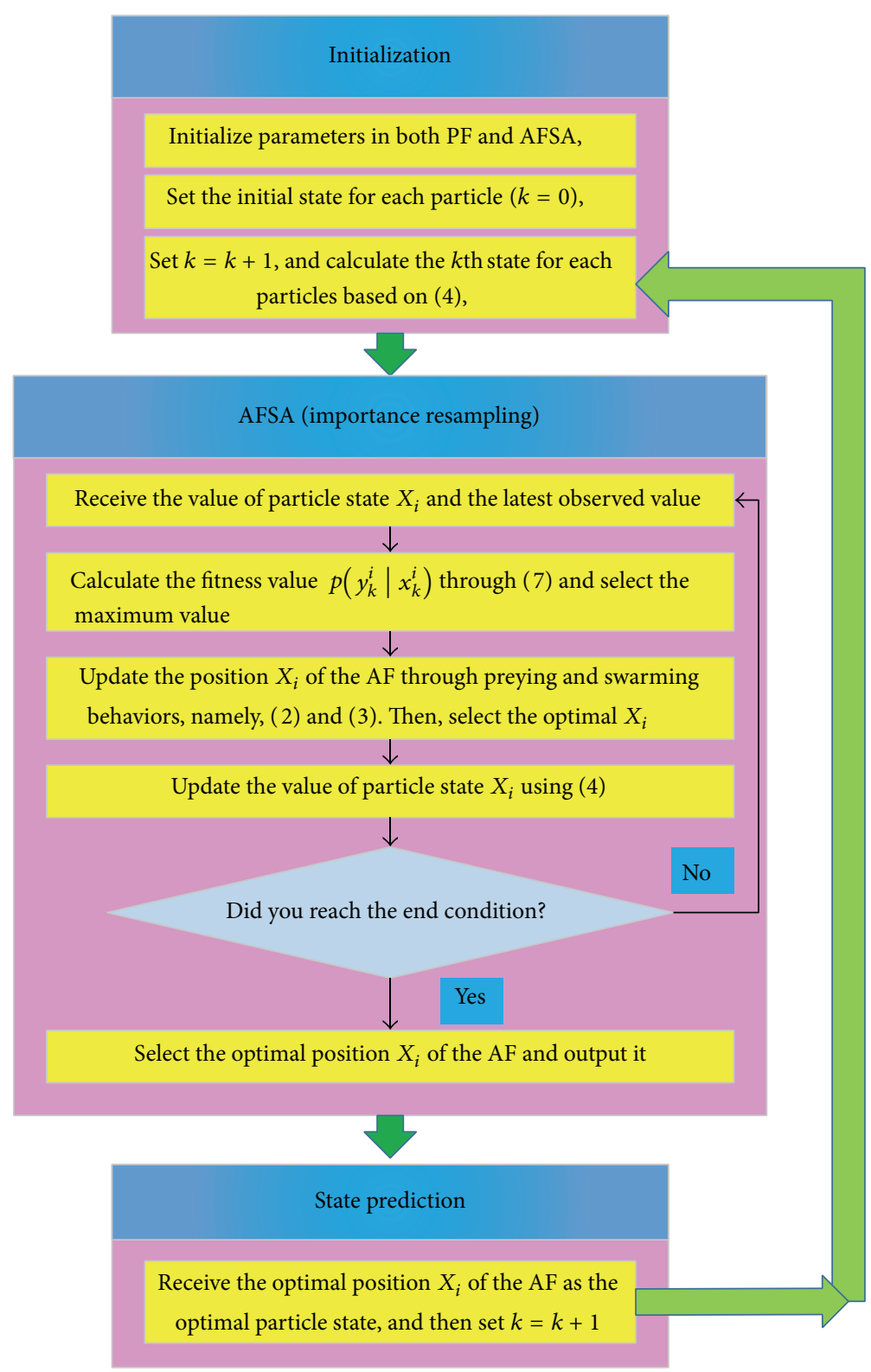

FIGURE 1: The operation process of AFSA-PF.

(2) Importance Resampling. The latest observed value is input to the AFSA, together with the corresponding particle state $X_{i}$ that acts as the initial position of the AF. The fitness value $p\left(y_{k}^{i} \mid x_{k}^{i}\right)$, calculated using (7), acts as the objective function $Y$ in (2).

The position $X_{i}$ of each AF is continuously updated based on the preying and swarming behaviors, as described in (2) and (3). The iteration process terminates once a predetermined iteration number is attained or the variation of the AF is less than a specified threshold.

(3) State Prediction. After the update process for all the AFs, the optimal position $X_{i}$ can be obtained, which is input to (4) as $x_{k-1}$ to acquire the next particle state $x_{k}$. Then, we set $k=k+1$ and repeat Steps (2 3) [19].
In AFSA-PF, $\mathrm{PF}$ is responsible for providing new observed values to the state estimate, and the AFSA is in charge of driving the particles toward the high-likelihood areas, which solves the problem that only a small fraction of the particles are in the high-likelihood areas and thus maintaining the diversity of the particles.

In order to explain the process of AFSA-PF more intuitively, Figure 1 is shown below.

\section{RUL Prediction for Li-Ion Batteries}

To avoid the phenomenon of particle degeneracy and obtain a predicted result with better accuracy and higher precision, we adopt AFSA-PF. As mentioned before in the Introduction, $\mathrm{RPF}$ is an effective method to avoid the degeneracy; thus, in 


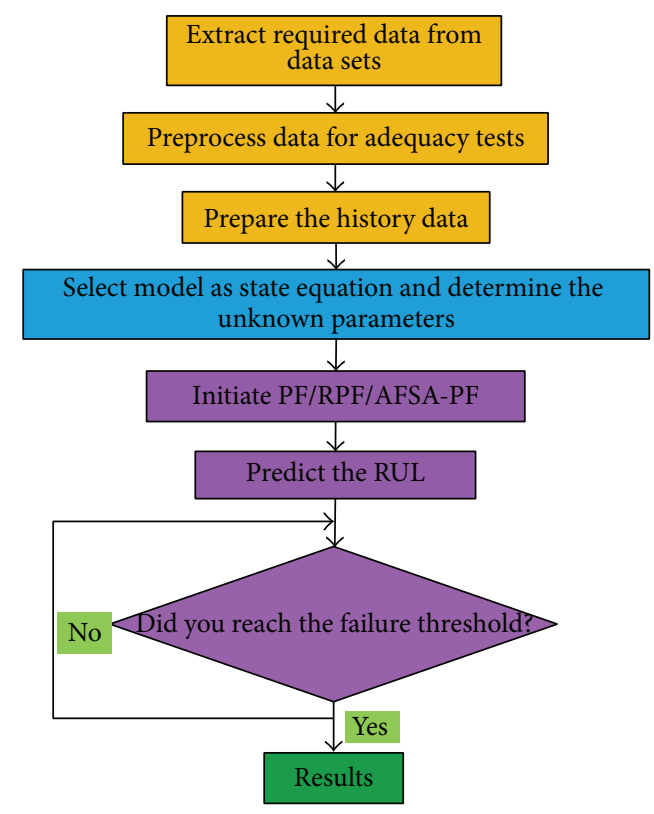

FIGURE 2: Flow of RUL prediction for Li-ion batteries.

this study, we compare AFSA-PF and RPF based on accuracy and precision. Based on the principle of $\mathrm{PF}$, a state equation for Li-ion batteries is required in both AFSA-PF and RPF. However, a number of unknown parameters always exist in the state equation to be estimated. Although PF can update the parameters when predicting the RUL, this momentary updating of parameters is extremely time-consuming, which impedes the popularization of AFSA-PF and RPF in engineering applications. Moreover, the objects we predict are almost degenerated over time; thus, a considerable amount of history data could be accumulated under normal conditions. Given this condition, by applying a number of history data, we can determine the unknown parameters before predicting RUL using AFSA-PF or RPF to obtain time-saving predictions without updating parameters momentarily. The procedures of the experiments for predicting the RUL of Liion batteries are shown in Figure 2.

The detailed descriptions are as follows.

First step is the data preprocessing. An appropriate health indicator is selected and the failure threshold is determined. The data selected for verifying the effectiveness of AFSA-PF and RPF should be sufficient to surpass the failure threshold. Here, we select the capacity of Li-ion batteries as the health indicator.

Second, an appropriate state equation is ensured to be available.

Third, based on the analysis of the data, part of the whole data is truncated to be the history data which are used to determine the unknown parameters in the state equation.

Fourth, the parameters in PF, RPF, and AFSA-PF are set, such as the number of particles, the covariance of the process noise, and the covariance of the measurement noise.

Finally, the RUL of the Li-ion batteries is predicted using PF, RPF, and AFSA-PF, and the results of these methods are then compared.

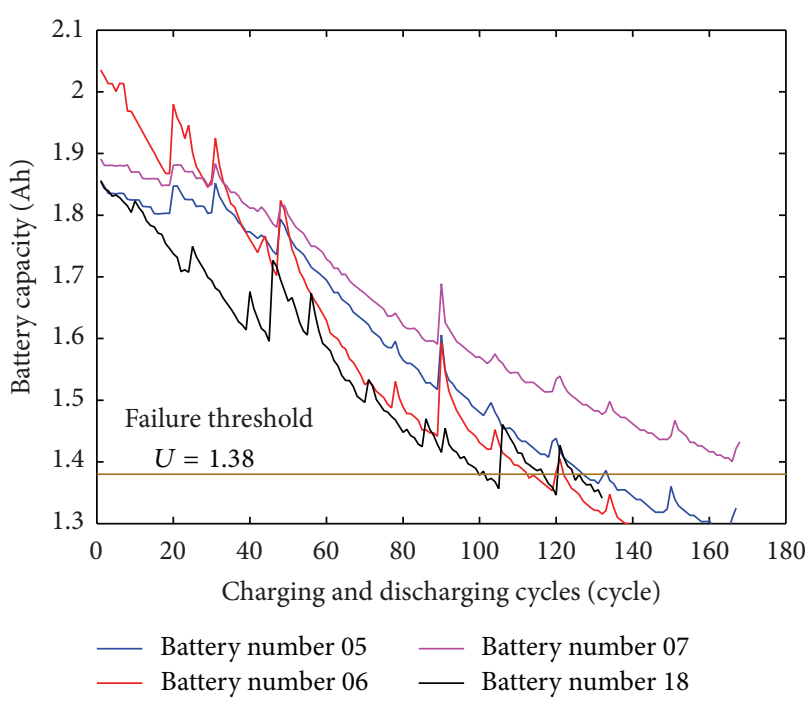

FIGURE 3: Capacity degradation curves for four Li-ion batteries in NASA PCoE.

3.1. Li-Ion Battery Data Set. To verify the performance of the AFSA-PF algorithm in predicting the RUL of Li-ion batteries, we adopt the Li-ion batteries data set from NASA AMES Center $[5,25]$. The battery data set is from the NASA PCoE. The Li-ion batteries are tested under certain conditions (with the temperature $+23^{\circ} \mathrm{C}$ ) and the test ending life is set as $70 \%$ of the rated capacity to measure the capacity degradation. Detailed information about the data set can be found in [26]. In this study, the capacity is considered as the health indicator of the degradation of the Li-ion battery. We set $70 \%$ of the rated capacity as the failure threshold $[27,28]$. For the selected data set, the rated capacity is $2 \mathrm{Ah}$ and the failure threshold $U$ is set as $1.38 \mathrm{Ah}$, which means that when the capacity degenerates to $1.38 \mathrm{Ah}$, the experiment will be stopped.

The data set includes the capacity of four batteries, that is, Batteries number 05, number 06, number 07, and number 18 . The Li-ion battery capacity degradation is shown in Figure 3.

Figure 3 shows that Battery number 07 cannot reach the failure threshold during the known cycles; thus, we reject the data for Battery number 07. Hereinafter, we will only discuss Batteries number 05, number 06, and number 18.

3.2. Empirical Degradation Model of Li-Ion Batteries. To apply AFSA-PF, a state equation is in need. Numerous models have been proposed for Li-ion batteries, such as Gaussian process regression (GPR) model [29], exponential degradation model $[30,31]$, and polynomial model [31]. However, some of these models are independent of the physical structure of Li-ion batteries and simply reflect the degradation process. The empirical degradation model, which is proposed by Saha and Goebel and derived from the physical mechanism of Li-ion batteries [2], adequately considers the influences of different operational modes (charge, discharge, and rest) on the capacity. Therefore, we conduct the battery capacity prediction based on this model in this study. 
The empirical degradation model can be described by the following equation:

$$
C_{k+1}=\eta_{C} C_{k}+\beta_{1} \exp \left(-\frac{\beta_{2}}{\Delta t_{k}}\right),
$$

where $C_{k}$ denotes the charge capacity of the $k_{\text {th }}$ cycle, $\eta_{C}$ denotes the coulombic efficiency, $\beta_{1}$ and $\beta_{2}$ denote the unknown parameters to be estimated, and $\Delta t_{k}$ denotes the rest period between cycles $k$ and $k+1$. In this study, we set $\eta_{C}=0.997[26]$ and $\Delta t_{k}=1$.

\subsection{Parameter Settings for Predicting the RUL of Li-Ion} Batteries. To integrate the model-based method with the data-driven method, an empirical degradation model is introduced as (8), and the unknown parameters, $\beta_{1}$ and $\beta_{2}$, are identified using $\mathrm{PF}$, which is an efficient method to estimate unknown parameters in nonlinear physical models with non-Gaussian noise [32]. Theoretically, the unknown parameters can be real-time updated using PF as a part of the prognostic process. However, in real engineering applications, the capacity degradation of Li-ion batteries is a gradual decay process, and the model parameters change slowly during the full life cycle. Therefore, it seems to be time-consuming if a real-time updating of the unknown parameters based on history data is adopted. In this study, we use the history data to identify the parameters before the AFSA-PF prediction instead of real-time parameter updating. The detailed real-time parameter updating process can refer to [32].

To avoid the occasionality of the validity of the experiments, we provide the RUL estimation results of Batteries number 05 , number 06 , and number 18 , which, respectively, contain 167, 168, and 132 data points, and the real-life times are 127, 112, and 100 cycles, respectively. Among the three batteries, the shortest life is 100 cycles. Thus, we consider the previous $40,50,60,70$, and 80 data samples, respectively, as the history data to determine the unknown parameters $\beta_{1}$ and $\beta_{2}$ and then to predict the RUL of the Li-ion batteries. To illustrate the effect of the proposed method without taking up too much space, we consider an example in which the unknown parameters are determined by the previous 60 data samples.

In the selected exemplificative experiment, we use the former 60 data samples of Battery number 05 to estimate the parameters. Given the randomness in the training algorithm, we typically cannot obtain exactly the same parameter values each time. Thus, in this study, we train the PF 25 times, and finally set the average of the whole estimated results, $\beta_{1}=$ -0.8 and $\beta_{2}=6$, as the determined parameters for future use. Given that the three batteries are of the same type and tested under the same conditions, we assume that the obtained state equation is suitable for all of them, which will be verified in the next section.

As mentioned above, the state equation is established using (8) with $\beta_{1}=-0.8$ and $\beta_{2}=6$; the observation equation is set as $Z_{k+1}=C_{k+1}+v_{k}$; and the objective function is set using (7). The parameters are set as follows: the number of particles $N=200$, the state initial values of the

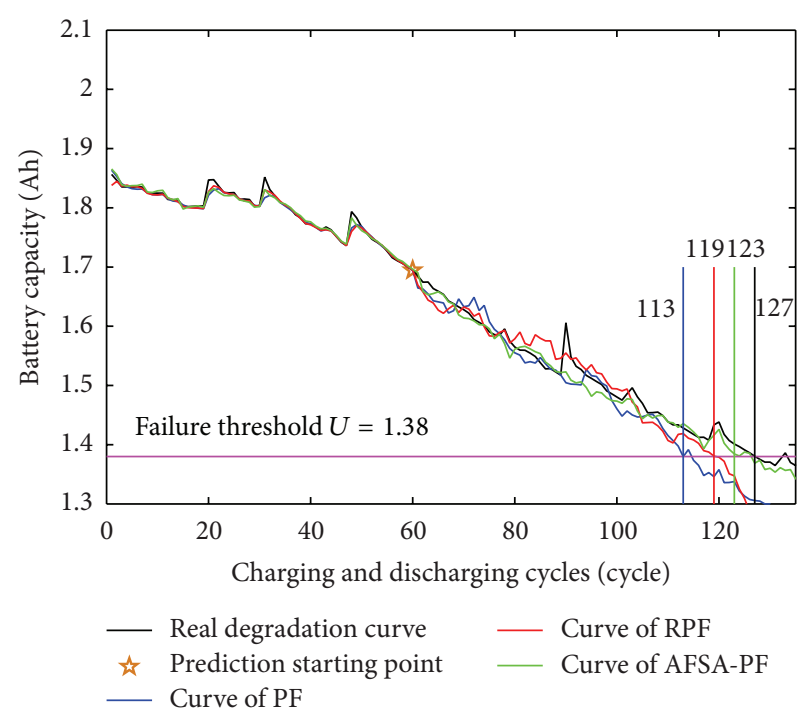

FIgURE 4: Battery RUL prediction results using basic PF, RPF, and AFSA-PF (Battery number 05).

battery capacity are the first data in the data set of Batteries number 05 , number 06 , and number 18 , the covariance of the process noise $w_{k} R=0.0001$, and the covariance of the measurement noise $v_{k} Q=0.0001$. Noise is considered as the Gaussian white noise to make the experiments close to the ideal situation. The parameters of the AFSA are set as follows: $\sigma_{v}^{2}=R=0.0001, s=0.00008, v=0.1, \delta=0.1$, the number of iterations is 50, and the number of the AF is 200 .

\section{Results and Discussion}

As mentioned in Section 3.3, after all the necessary parameters are set, we predict the RUL of Batteries number 05, number 06, and number 18 using PF, RPF, and AFSA-PF. The prediction results and the PDF distributions of the results are shown in Figures 4-9. In Figures 4, 6, and 8, the blue, red and green curves before the prediction starting point, respectively, represent the tracking curves of PF, RPF, and AFSA-PF. The tracking curves and the real degradation curve are almost coincident in all the three figures, which demonstrates the effectiveness of the applied empirical degradation model and that the parameters in the state equation determined using the data for Battery number 5 are suitable for the other batteries, which is of great importance in engineering applications.

To analyze the prediction results explicitly, we discuss the RUL prediction results and PDF distributions. Figures 4, 6, and 8 show that the green curve has the best RUL prediction result, which is generated by AFSA-PF. Then the red curve has the second-best result, which is generated by RPF. The blue curve generated by PF comes in last. A summary for the prediction results is shown in Table 1. By comparison, AFSA$\mathrm{PF}$ is obviously superior to PF and RPF, and RPF is better than $\mathrm{PF}$, which is consistent with the principles mentioned before.

As shown in Figures 5, 7, and 9, the distributions of AFSA$\mathrm{PF}$ prediction results are narrower and taller than both that 
TABLE 1: Prediction results of PF, RPF, and AFSA-PF.

\begin{tabular}{|c|c|c|c|c|}
\hline Battery number & Prediction method & Prediction result (cycle) & Absolute error (cycle) & Relative error \\
\hline \multirow{3}{*}{ Number 05} & $\mathrm{PF}$ & 113 & 14 & $11.02 \%$ \\
\hline & $\mathrm{RPF}$ & 119 & 8 & $6.30 \%$ \\
\hline & AFSA-PF & 123 & 4 & $3.15 \%$ \\
\hline \multirow{3}{*}{ Number 06} & $\mathrm{PF}$ & 97 & 15 & $13.40 \%$ \\
\hline & $\mathrm{RPF}$ & 105 & 7 & $6.25 \%$ \\
\hline & AFSA-PF & 110 & 2 & $1.79 \%$ \\
\hline \multirow{3}{*}{ Number 18} & $\mathrm{PF}$ & 86 & 14 & $14 \%$ \\
\hline & $\mathrm{RPF}$ & 92 & 8 & $8 \%$ \\
\hline & AFSA-PF & 97 & 3 & $3 \%$ \\
\hline
\end{tabular}
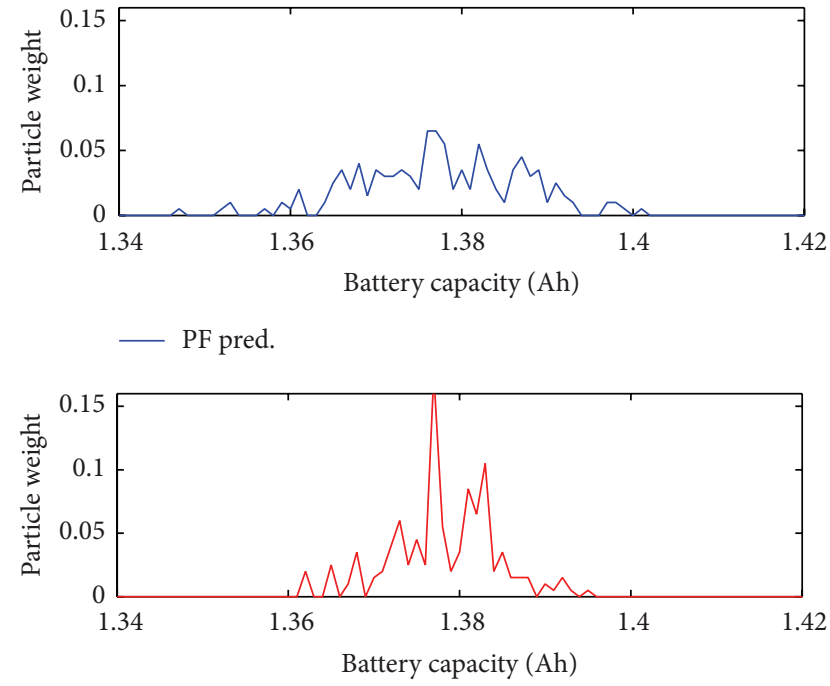

- RPF pred.

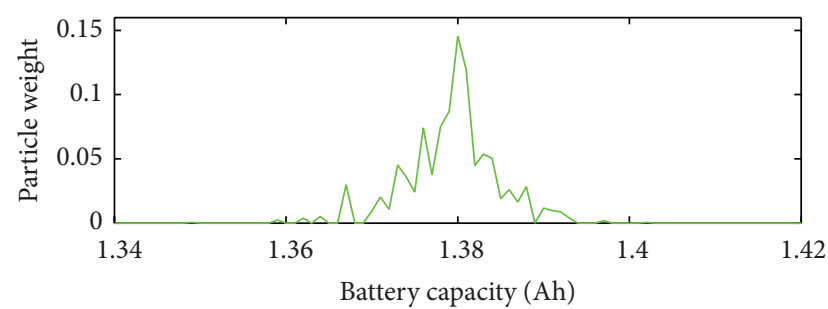

- AFSA-PF pred.

Figure 5: PDF distribution of the prediction result for Battery number 05 .

of PF and that of RPF, and RPF has better results than PF. The distributions of the prediction results show the precision of prediction. The narrower and taller the distributions are, the higher precision the results have. Thus, AFSA-PF shows a higher precision than the other two algorithms. Meanwhile, $\mathrm{RPF}$ is still better than PF.

The results demonstrate that AFSA-PF is indeed an effective approach in predicting the RUL of the Li-ion batteries. As expected, we have obtained better results from AFSA-PF than PF, and RPF because in AFSA-PF, traditional resampling methods are replaced by the preying and swarming behaviors

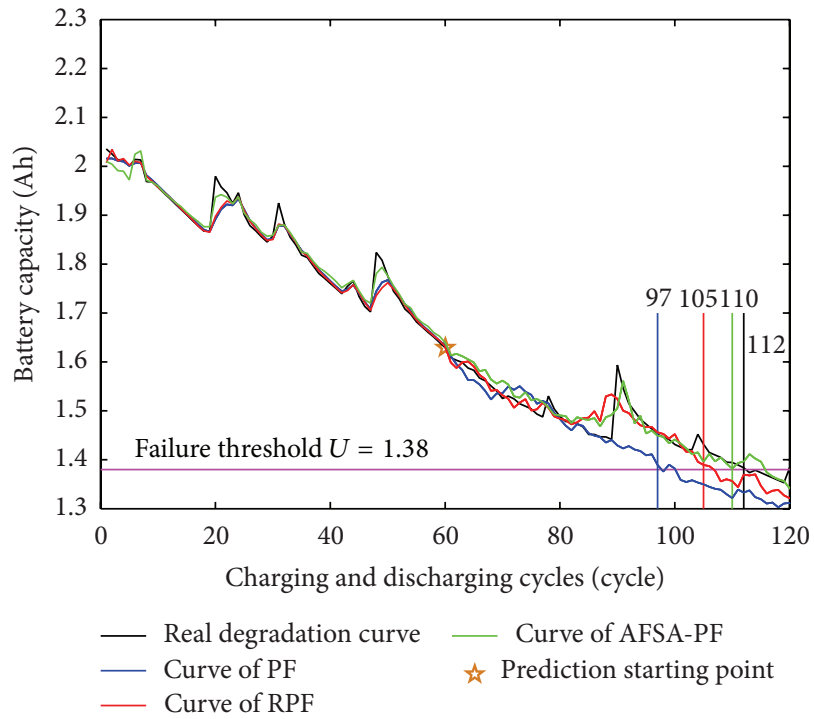

FIGURE 6: Battery RUL prediction results using basic PF, RPF, and AFSA-PF (Battery number 06).

of AFSA. In this approach, all the artificial fish make an utmost effort to find the optimal result freely, thereby driving the particles to the high-likelihood domain and in turn, improves the distribution of the particles and increases the accuracy and precision.

\section{Conclusions and Future Works}

This study uses an intelligent prognostic approach based on AFSA and PF to predict the RUL of Li-ion batteries. The main contribution of this research are as follows: (1) successfully applying AFSA-PF to predict the RUL of Li-ion batteries, (2) successfully illustrating the availability of combining model-based and data-driven methods in RUL prediction, and (3) in terms of predicting the RUL of Li-ion batteries, making a detailed comparison between AFSA-PF and RPF, as RPF is regarded as an effective method of preventing particle degeneracy. The experimental results indicate that the proposed AFSA-PF method is suitable for predicting the 


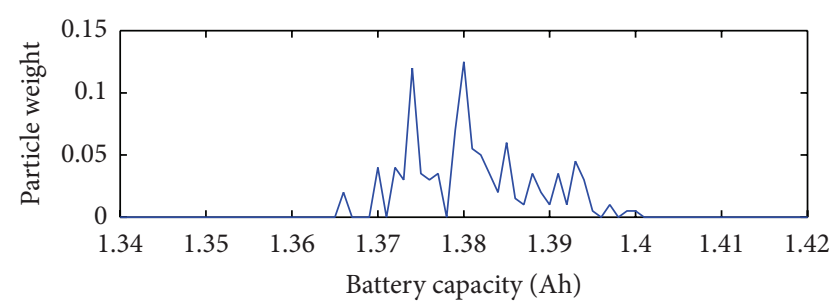

- PF pred.

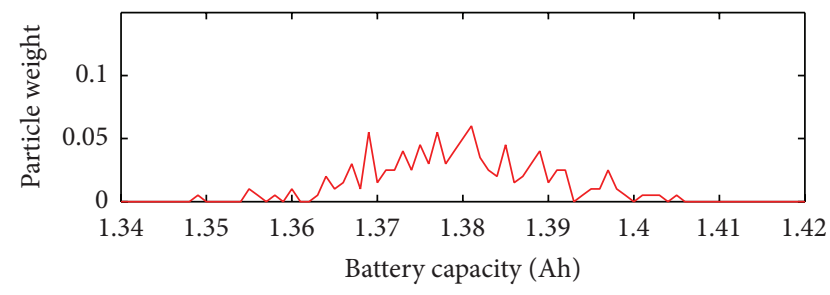

- RPF pred.

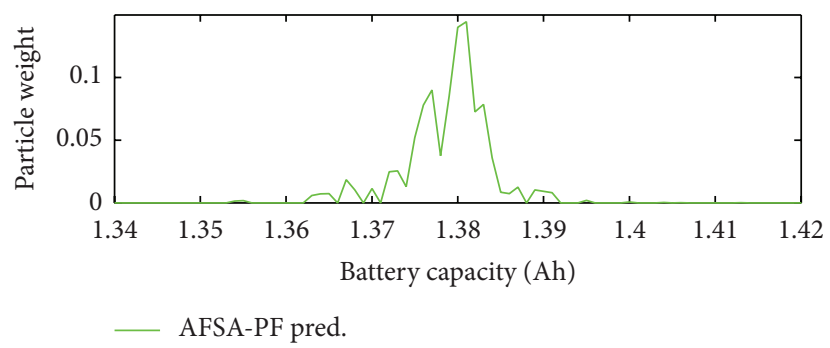

FIGURE 7: PDF distribution of the prediction result for Battery number 06 .

RUL of Li-ion batteries. Compared with PF and RPF, AFSA$\mathrm{PF}$ is more accurate and precise. The proposed method shows promising application in other kinds of batteries.

Our future research will focus on two problems. First, in the study, we noticed that the parameters in AFSA have a major influence on optimization. Selecting appropriate parameters for different optimization functions under different required precisions is difficult yet vitally important. Second, more research should be carried on solving the time-consuming problem in real-time prediction, because the introduction of intelligent thought increases algorithm complexity; moreover, how to realize real-time updating parameters without consuming too much resource is a challenge. AFSA itself is a rapid optimization method, but when combined with $\mathrm{PF}$, the speed is slowed down near to that of RPF, which limits the application of both AFSA-PF and RPF in engineering. Fortunately, the AFSA method is so flexible that it can be improved for better timeliness.

\section{Conflict of Interests}

The authors declare that there is no conflict of interests regarding the publication of this paper.

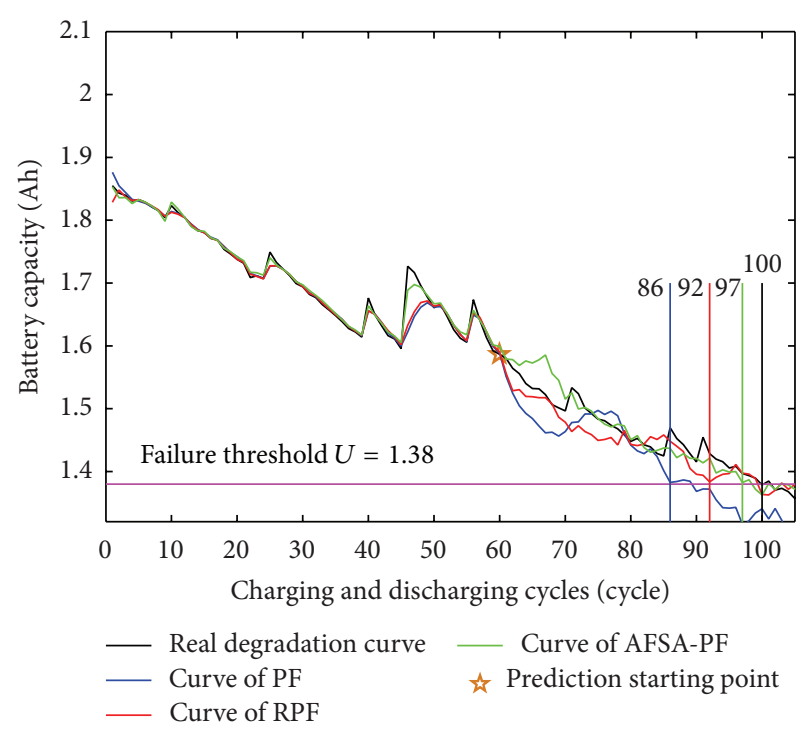

FIGURE 8: Battery RUL prediction results using basic PF, RPF, and AFSA-PF (Battery number 18).

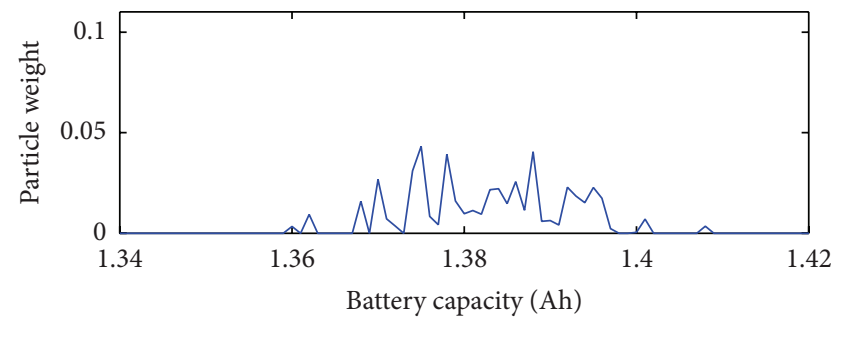

— PF pred.

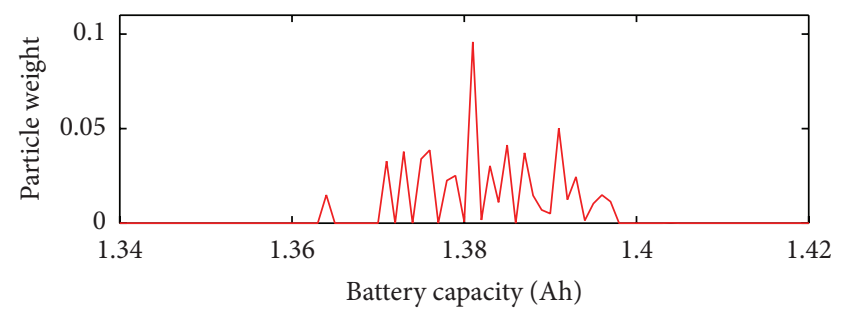

- RPF pred.

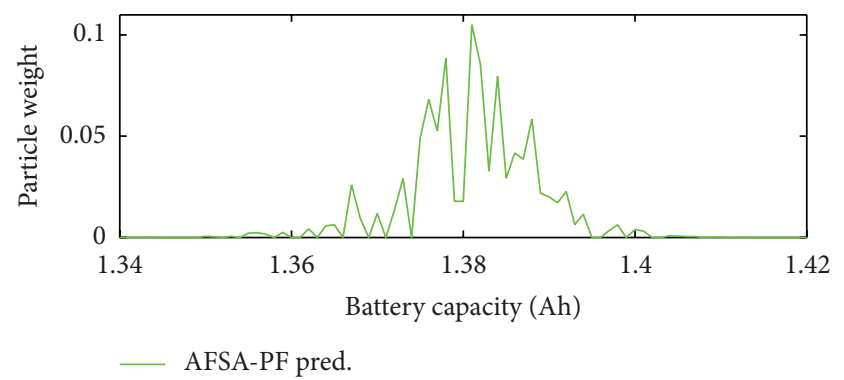

FIGURE 9: PDF distribution of the prediction result for Battery number 18. 


\section{Acknowledgments}

This research is supported by the National Natural Science Foundation of China (Grants nos. 61074083 and 51105019) and the Technology Foundation Program of National Defense (Grant no. Z132013B002).

\section{References}

[1] K. Goebel, B. Saha, A. Saxena, J. R. Celaya, and J. P. Christophersen, "Prognostics in battery health management," IEEE Instrumentation and Measurement Magazine, vol. 11, no. 4, pp. 33-40, 2008.

[2] B. Saha and K. Goebel, "Modeling li-ion battery capacity depletion in a particle filtering framework," in Proceedings of the Annual Conference of the Prognostics and Health Management Society, 2009.

[3] S.-W. Eom, M.-K. Kim, I.-J. Kim, S.-I. Moon, Y.-K. Sun, and H.S. Kim, "Life prediction and reliability assessment of lithium secondary batteries," Journal of Power Sources, vol. 174, no. 2, pp. 954-958, 2007.

[4] F. Rufus Jr., S. Lee, and A. Thakker, "Health monitoring algorithms for space application batteries," in Proceedings of the International Conference on Prognostics and Health Management (PHM '08), IEEE, October 2008.

[5] B. Saha, K. Goebel, and J. Christophersen, "Comparison of prognostic algorithms for estimating remaining useful life of batteries," Transactions of the Institute of Measurement and Control, vol. 31, no. 3-4, pp. 293-308, 2009.

[6] X. S. Si, W. Wang, C. H. Hu, and D. H. Zhou, "Remaining useful life estimation-a review on the statistical data driven approaches," European Journal of Operational Research, vol. 213, no. 1, pp. 1-14, 2011.

[7] J. Liu, A. Saxena, K. Goebel, B. Saha, and W. Wang, "An adaptive recurrent neural network for remaining useful life prediction of lithium-ion batteries," in Proceedings of the Annual Conference of the Prognostics and Health Management Society, 2010.

[8] J. Zhang and J. Lee, "A review on prognostics and health monitoring of Li-ion battery," Journal of Power Sources, vol. 196, no. 15, pp. 6007-6014, 2011.

[9] B. Saha, K. Goebel, S. Poll, and J. Christophersen, "Prognostics methods for battery health monitoring using a Bayesian framework," IEEE Transactions on Instrumentation and Measurement, vol. 58, no. 2, pp. 291-296, 2009.

[10] B. Saha, K. Goebel, S. Poll, and J. Christophersen, "An integrated approach to battery health monitoring using bayesian regression and state estimation," in Proceedings of the 2007 IEEE Autotestcon, pp. 646-653, September 2007.

[11] E. Zio and G. Peloni, "Particle filtering prognostic estimation of the remaining useful life of nonlinear components," Reliability Engineering and System Safety, vol. 96, no. 3, pp. 403-409, 2011.

[12] A. Doucet, N. J. Gordon, and V. Krishnamurthy, "Particle filters for state estimation of jump Markov linear systems," IEEE Transactions on Signal Processing, vol. 49, no. 3, pp. 613-624, 2001.

[13] M. S. Arulampalam, S. Maskell, N. Gordon, and T. Clapp, "A tutorial on particle filters for online nonlinear/non-Gaussian Bayesian tracking," IEEE Transactions on Signal Processing, vol. 50, no. 2, pp. 174-188, 2002.

[14] E. A. Wan and R. van der Merwe, "The unscented Kalman filter for nonlinear estimation," in Proceedings of the IEEE Adaptive
Systems for Signal Processing, Communications, and Control Symposium (AS-SPCC '00), pp. 153-158, Lake Louise, Canada, October 2000.

[15] C. Musso, N. Oudjane, and F. Le Gland, "Improving regularised particle filters," in Sequential Monte Carlo Methods in Practice, pp. 247-271, Springer, New York, NY, USA, 2001.

[16] Y. Gao, S. Gao, and Y. Gu, "Improved regularized particle filter algorithm for SINS/SAR integrated navigation," in Proceedings of the International Conference on Computer Application and System Modeling (ICCASM '10), vol. 9, pp. V9-70-V9-74, Taiyuan, China, October 2010.

[17] J. Liu, W. Wang, and F. Ma, "A regularized auxiliary particle filtering approach for system state estimation and battery life prediction," Smart Materials and Structures, vol. 20, no. 7, Article ID 075021, 2011.

[18] T. R. Lu, L. Zhu, and D. F. Li, "Swarm intelligence algorithm for particle filtering," Journal of Xidian University, vol. 35, no. 3, pp. 273-277, 2007.

[19] Y. Tian and L. Chen, "Unscented particle filter algorithm based on artificial fish swarm algorithm," in Proceeding of the 8th International Conference on Natural Computation (ICNC '12), pp. 1123-1126, Chongqing, China, May 2012.

[20] X. L. Li, Z. J. Shao, and J. X. Qian, "Optimizing method based on autonomous animats: fish-swarm Algorithm," System Engineering Theory and Practice, vol. 22, no. 11, pp. 32-38, 2002.

[21] J. W. Ma, G. L. Zhang, and H. Xie, "By using artificial fish swarm algorithm optimization of feed-forward neural networks," Computer Application, vol. 24, no. 10, pp. 21-23, 2004.

[22] R. Li and Q. Liu, "Particle swarm optimization based on Unscented particle filter algorithm," Computer Engineering, vol. 13, no. 37, pp. 153-155, 2011.

[23] L. Jiye, C. Xihong, L. Qiang, and S. Jizhe, "Prediction of satellite clock errors using LS-SVM optimized by improved artificial fish swarm algorithm," in Proceedings of the IEEE International Conference on Signal Processing, Communication and Computing (ICSPCC '13), KunMing, China, 2013.

[24] N. J. Gordon, D. J. Salmond, and A. F. M. Smith, "Novel approach to nonlinear/non-gaussian Bayesian state estimation," IEE Proceedings F: Radar and Signal Processing, vol. 140, no. 2, pp. 107-113, 1993.

[25] J. Y. Liu, X. H. Chen, Q. Liu, and J. Z. Sun, Battery Data Set, NASA Ames Prognostics Data Repository, NASA Ames, Moffett Field, Calif, USA, 2007.

[26] D. Liu, Y. Luo, L. Guo, and Y. Peng, "Uncertainty quantification of fusion prognostics for lithium-ion battery remaining useful life estimation," in Proceedings of the IEEE Conference on Prognostics and Health Management (PHM '13), pp. 1-8, Gaithersburg, Md, USA, June 2013.

[27] M. Dubarry and B. Y. Liaw, "Identify capacity fading mechanism in a commercial $\mathrm{LiFePO}_{4}$ cell," Journal of Power Sources, vol. 194, no. 1, pp. 541-549, 2009.

[28] IEEE Std.1188-2005 IEEE Recommended Practices for Maintenance, Testing and Replacement of Valve Regulated Lead-Acid (VRLA) Batteries in Stationary Applications.

[29] D. Liu, J. Pang, J. Zhou, Y. Peng, and M. Pecht, "Prognostics for state of health estimation of lithium-ion batteries based on combination Gaussian process functional regression," Microelectronics Reliability, vol. 53, no. 6, pp. 832-839, 2013.

[30] Z. Fan, G. Liu, X. Si et al., "Degradation data-driven approach for remaining useful life estimation," Journal of Systems Engineering and Electronics, vol. 24, no. 1, pp. 173-182, 2013. 
[31] Y. Xing, E. W. M. Ma, K. Tsui, and M. Pecht, "An ensemble model for predicting the remaining useful performance of lithium-ion batteries," Microelectronics Reliability, vol. 53, no. 6, pp. 811-820, 2013.

[32] D. An, J.-H. Choi, and N. H. Kim, "Prognostics 101: a tutorial for particle filter-based prognostics algorithm using Matlab," Reliability Engineering \& System Safety, vol. 115, pp. 161-169, 2013. 


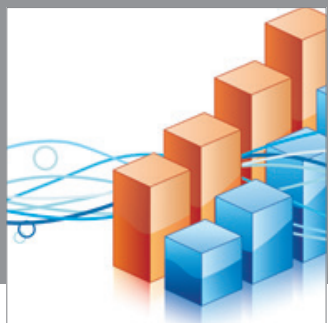

Advances in

Operations Research

mansans

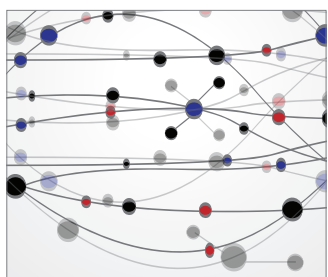

The Scientific World Journal
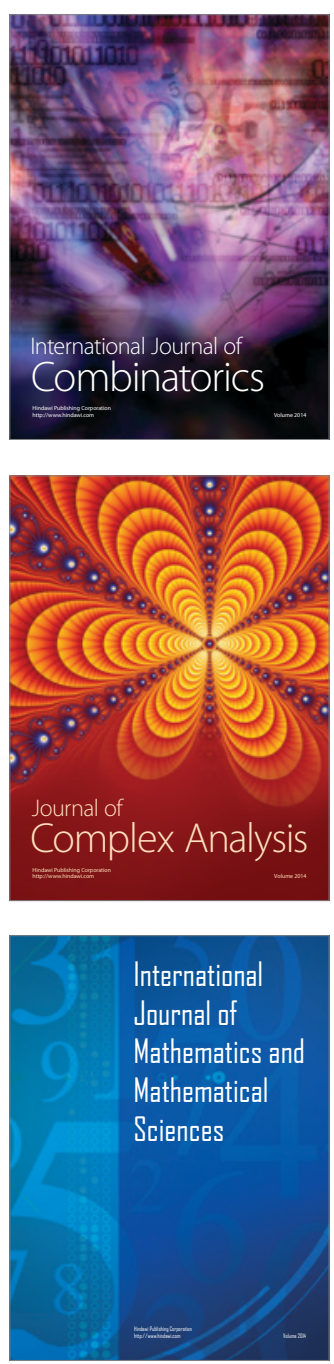
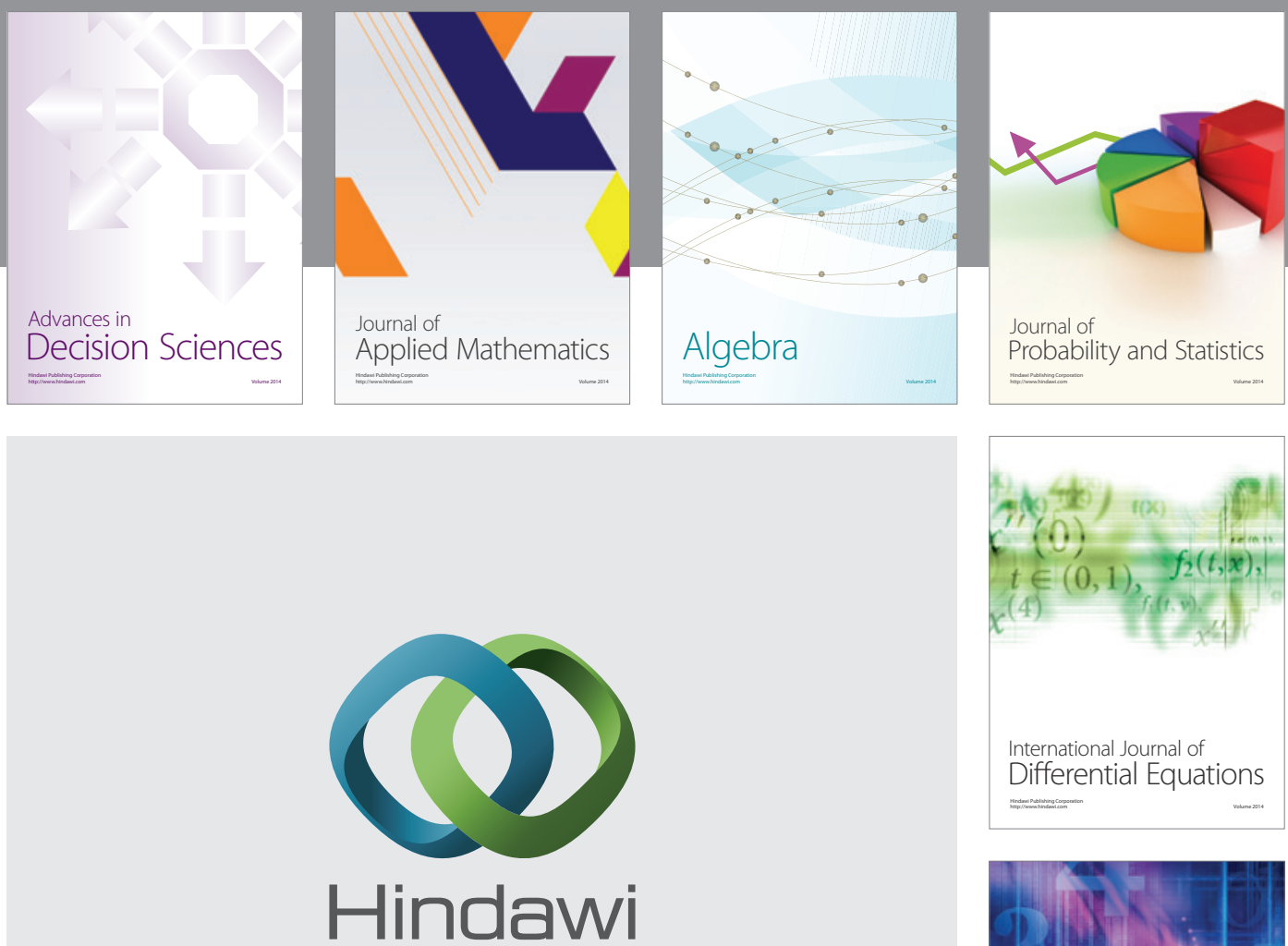

Submit your manuscripts at http://www.hindawi.com
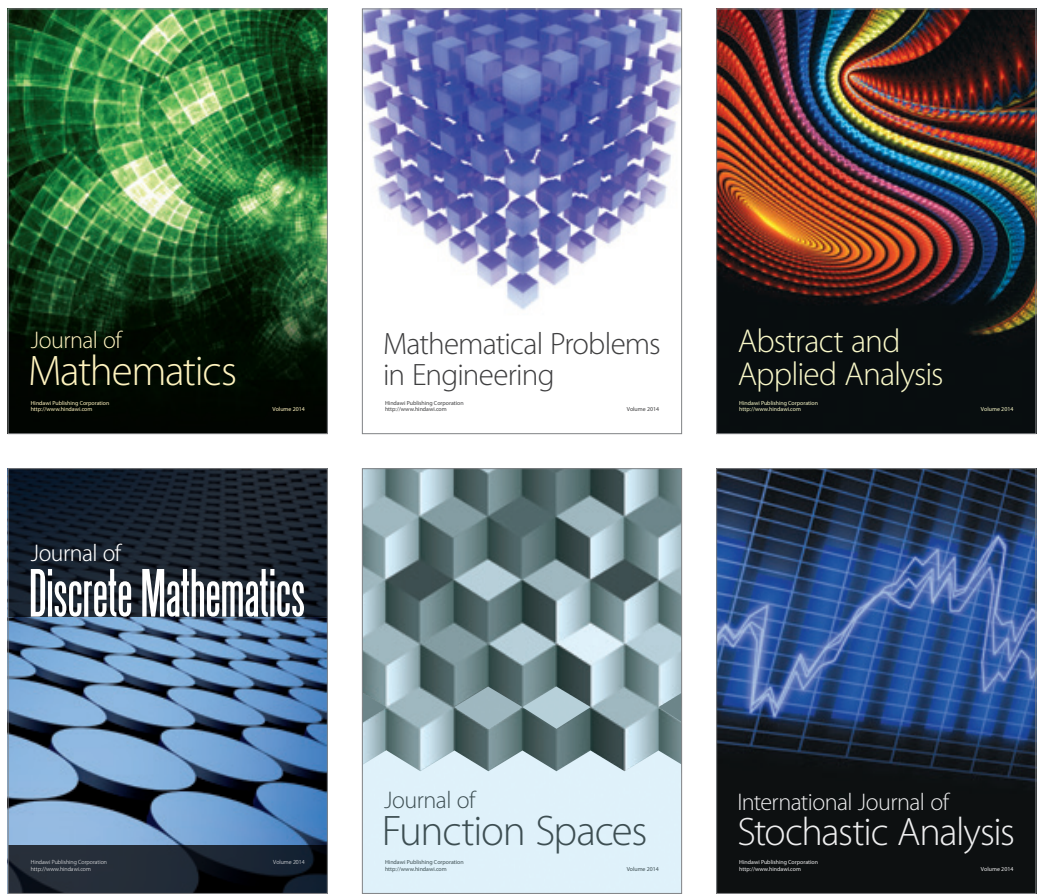

Journal of

Function Spaces

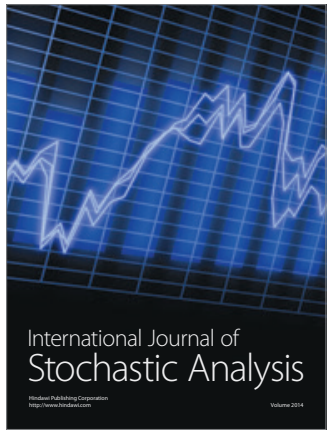

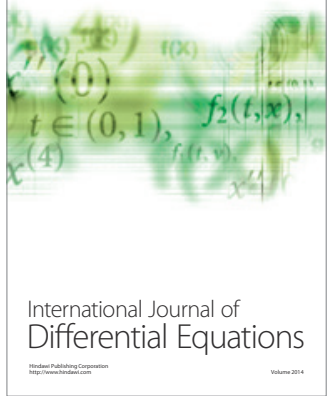
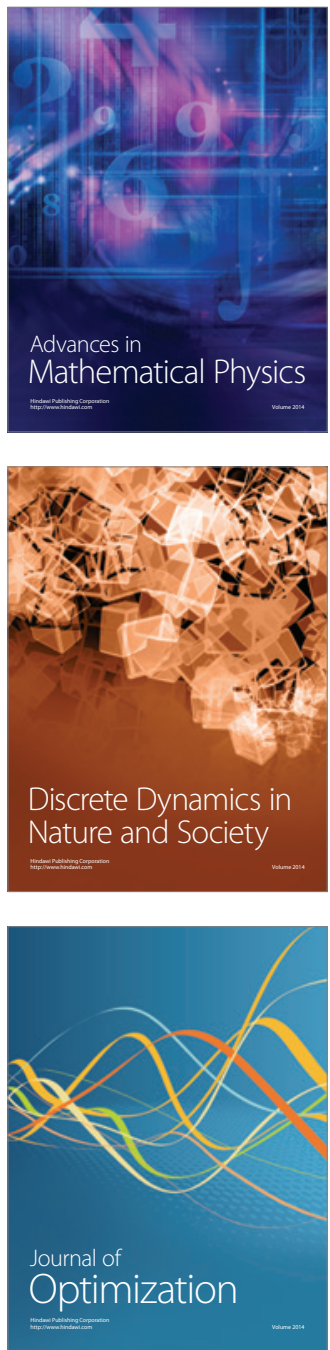